\title{
Analytisches Inhaltsverzeichnis.
}

I. Kapitel.

Begriffsbestimmungen.

1. Rejehtum und Austausch $\ldots \ldots \ldots \ldots \ldots \ldots \ldots \ldots \ldots \ldots \ldots \ldots \ldots \ldots \ldots, 1$

2. Austauschbare Güter $\ldots \ldots \ldots \ldots \ldots \ldots \ldots \ldots \ldots \ldots \ldots \ldots \ldots \ldots \ldots \ldots, 3$

\$ 3. Umlaut des Geldes gegen Güter $\ldots \ldots \ldots \ldots \ldots \ldots \ldots \ldots \ldots \ldots \ldots \ldots \ldots$ ธ

II. Kapitel.

Beziehungen der Kaufluaft des Geldes zur Verkehrsgleichung.

1. Die verschiedenen Zirkulationsmittel $\ldots \ldots \ldots \ldots \ldots \ldots \ldots \ldots \ldots \ldots, 7$

5 2. Die Verkehrsgleichung artthmetisch ausgedrückt ................... 11

8. Die Verkehrsgleichung mechanisch ausgedruickt $\ldots \ldots \ldots \ldots \ldots \ldots \ldots \ldots, 17$

4. Die Verkehrsgleichung algebraisch ausgedrückt ................... 19

§ 5. SchluBfolgerung und Erläuterungen............................... 23

\section{Kapitel.}

Einflub der Depositenumlaufsmittel auf die Gleichung und infolgedessen auf die Kaufkraft.

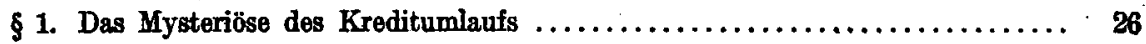

\$ 2. Die Basis des Kreditumlaufs $\ldots \ldots \ldots \ldots \ldots \ldots \ldots \ldots \ldots \ldots \ldots \ldots \ldots \ldots, 32$

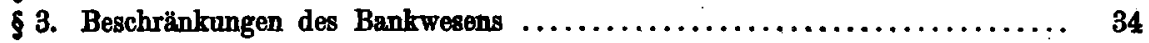

\$ 4. Revision der Verkehrsgloichung $\ldots \ldots \ldots \ldots \ldots \ldots \ldots \ldots \ldots \ldots \ldots \ldots, 38$

5. Depositenurnlaufsmittel in ihrem normalen Verhaltnis zam Gelde ......... 40

§ 6. Zusammenfassung $\ldots \ldots \ldots \ldots \ldots \ldots \ldots \ldots \ldots \ldots \ldots \ldots \ldots \ldots, 43$

IV. Kapitel.

Störung der Gleichung und der Kaufkraft in Perioden des Utbergangs.

§ 1. Langsamkeit der Zinsadjustierung an die Preisbewegungen ............. 44

§ 2. Wie ein Steigen der Preise ein weiteres Steigen nach sich zieht ......... 47

8 3. Umfang der Störungen in der Gleichung $\ldots \ldots \ldots \ldots \ldots \ldots \ldots \ldots \ldots \ldots$. 49

§. Wie ein Steigen der Preise in einer Krisis seinen Höhepunkt erreicht ...... 61

5. Vollendung des Kreditzykdus ................................. 54

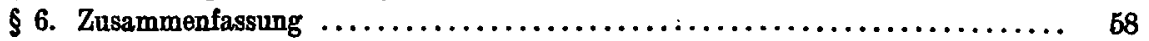


\$ 1. Einflub der Produktions- und Konsuntionsbedingangen auf den Handel und

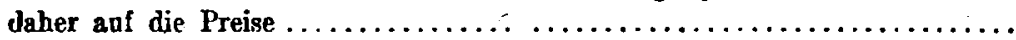

§2. EinfluB der Beziohungen zwisohen Prodnzenten und Konsumenten ant den

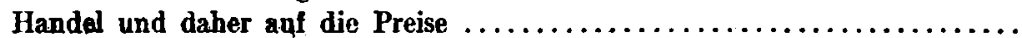

8 3. Einfluß persönlicher Gowohnheiten auf die Umianfsgeschwindigkeit und daher

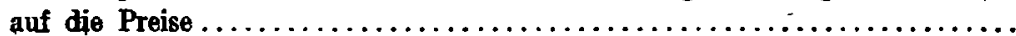

§ 4. EinftaB der Zahlengssysteme auf die Umiaufsgesehwindigkeit und daher auf

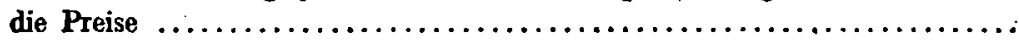

8. EinfluB allgemeiner Ursechen anf die Umlaufsgesobwindigkeit und daher auf

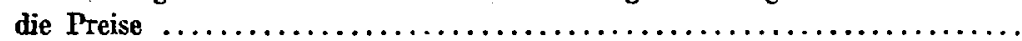

§ 6. Einflüsse auf den Umfang der dem Scheciverkehr unterworfenen Depositen

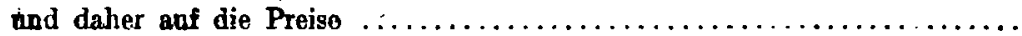

VI. Kapitel.

\section{Indirekto Einwirkangen (Fortsetzung).}

\$1. Binfluß des AuBerhandels auf die Geldmenge und daher auf die Preise .....

\$2. BinfluB des Schmelzens nnd Ausmuinzens auf die Geldmenge und daher anf

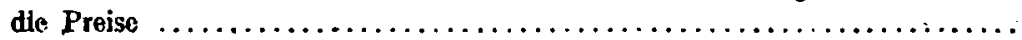

§ 3. EinfluB der Produktion und Konsumtion der Geldmetalle auf die Geldmenge

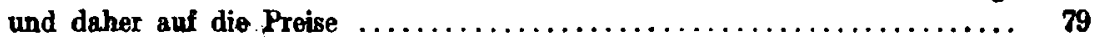

§ 4. Mechanische Darstallung dieset Einflüsse $\ldots \ldots \ldots \ldots \ldots \ldots \ldots \ldots \ldots, 84$

VII. Kapitel.

Einwirkung der Geldsysteme auf die Kaufluaft.

§ 1. Das Greshamsohe Gesetz $\ldots \ldots \ldots \ldots \ldots \ldots \ldots \ldots \ldots \ldots \ldots \ldots \ldots \ldots$ 89

\$ 2. Fälle, in denen der Bimetallismus sofort versagt .................. 91

\$3. Fäle, in denen der Bimetallismus versagt, nachdem die Produktion die Konsumtion überholt hat $\ldots \ldots \ldots \ldots \ldots \ldots \ldots \ldots \ldots \ldots \ldots \ldots \ldots \ldots, \quad 97$

\$ 4. Die hinkonde Wührung; die Golddevisenwăhrung ................. 101

\$ 6. Der Bimetallismus in Frankreich $\ldots \ldots \ldots \ldots \ldots \ldots \ldots \ldots \ldots \ldots \ldots \ldots, 105$

§ 6. Lehren aus dem französischen Experiment ........................ 108

8. Die hinkende Währung in Indien $\ldots \ldots \ldots \ldots \ldots \ldots \ldots \ldots \ldots \ldots \ldots, 110$

8. Die hinkende Whang in den Vereinigten Stanten ................. 112

8. Allgemeine Beschreibung des Systems in den Vereinigten Staaten ....... 114

VIII. Kapitel.

Einwirkung der Geldquantităt und anderer Faktoron auf die Kaufkraft nnd ihre Weohselwirkung.

\$1. Die Verkehrsgleichung drickt keinen kausalen Zusammenhang 2wischen ihren Gliedern ans $\ldots \ldots \ldots \ldots \ldots \ldots \ldots \ldots \ldots \ldots \ldots \ldots \ldots \ldots \ldots \ldots, 118$

§ 2. Wirkungen einer Veränderung im Gelde (G). Die Quantitätstheorie in kausalem

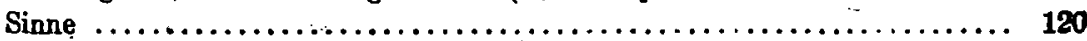




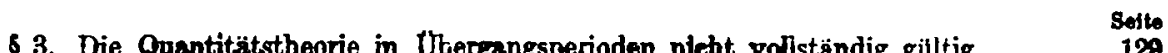

5 4. Wirkungen einer Veränderung in den Depositen $\left(G^{\prime}\right)$ in Beziehung sum

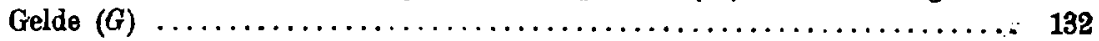

$\S$ 5. Wirkungen ron Veränderungen in der Umlaufsgeschwindigkeit $\left(U\right.$ und $\left.U^{\prime}\right) \ldots \quad 134$

\$6. Wirkungen von Veränderungen im Handelsvolumen (in den Q) ....... 134

§ 7. Kann das Preisniveau als Ursache und als Wirkung betrachtet werden? ... 138

\$ 8. Unterschoidung awischen der Verursachung individueller Preise und dem Preisniveau $\ldots \ldots \ldots \ldots \ldots \ldots \ldots \ldots \ldots \ldots \ldots \ldots \ldots \ldots \ldots \ldots \ldots \ldots \ldots \ldots$

§. Zusammenfassung $\ldots \ldots \ldots \ldots \ldots \ldots \ldots \ldots \ldots \ldots \ldots \ldots \ldots \ldots \ldots \ldots$

IX. Kapitel.

Die Zerstrouung der Preise macht eino Indexmummer für die Kanfkraft erforderlich.

\$1. Einige Preise reagieren nur schwerfallig auf allgemeine Preisbewegangen ... 149

6 2. Infolgedessen müssen andere Preise überstark reagieren $\ldots \ldots \ldots \ldots \ldots \ldots \ldots$ 154

§ 3. Umformung der rechten Seito der Verkehrsgleichung von $\Sigma p Q$ in $P H \ldots \ldots$. 157

8. Zusammenfassung $\ldots \ldots \ldots \ldots \ldots \ldots \ldots \ldots \ldots \ldots \ldots \ldots \ldots \ldots \ldots$

\section{Kapitel.}

Die besten Indexnummern der Kaufkraft.

§ 1. Formen der Indexnummern ............................... 160

§. Die verschiedenen $Z \mathbf{Z w e c k e}$ der Indexnummern $\ldots \ldots \ldots \ldots \ldots \ldots \ldots \ldots, 165$

§ 8. Die Indexnummer als Norm für aufgeschobene Zablungen ............ 168

§ 4. Aufgeschobene Zahlungen auf dem Gesamtaustausch bernhend.......... 176

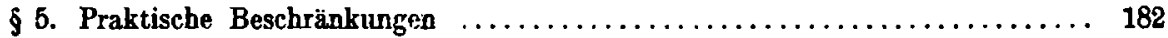

$\S 6$. Zusammenfassung $\ldots \ldots \ldots \ldots \ldots \ldots \ldots \ldots \ldots \ldots \ldots \ldots \ldots \ldots \ldots \ldots, 187$

\section{Kapitel.}

Statistischer Nachweis. Allgemeiner historischer Rückblick.

§ 1. Die leteten tausend Jahre $\ldots \ldots \ldots \ldots \ldots \ldots \ldots \ldots \ldots \ldots \ldots \ldots \ldots$

\$ 2. Die letzten vier Jahrhunderte $\ldots \ldots \ldots \ldots \ldots \ldots \ldots \ldots \ldots \ldots \ldots \ldots \ldots \ldots \ldots 1$

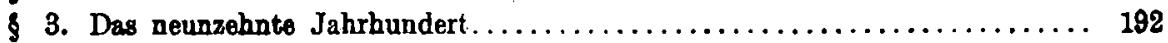

\$ 4. Seine fänf Preisberregungen $\ldots \ldots \ldots \ldots \ldots \ldots \ldots \ldots \ldots \ldots \ldots \ldots \ldots \ldots \ldots$

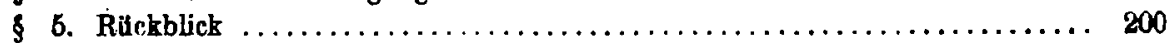

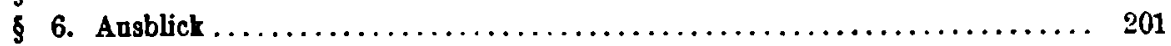

$\$$ 7. Papiergeld $\ldots \ldots \ldots \ldots \ldots \ldots \ldots \ldots \ldots \ldots \ldots \ldots \ldots \ldots \ldots \ldots \ldots \ldots$

8. Das Papiargeld in Frankreich $\ldots \ldots \ldots \ldots \ldots \ldots \ldots \ldots \ldots \ldots \ldots \ldots \ldots \ldots \ldots$

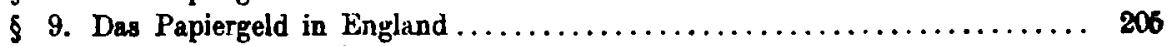

\$ 10. Das Papiergeld in Österreich $\ldots \ldots \ldots \ldots \ldots \ldots \ldots \ldots \ldots \ldots \ldots \ldots \ldots \ldots$

$\$$ 11. Frühamerikanisches Papiergeld $\ldots \ldots \ldots \ldots \ldots \ldots \ldots \ldots \ldots \ldots \ldots \ldots$

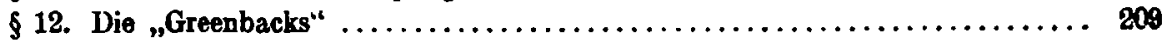

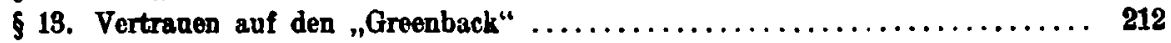

\$ 14. Das Papiergeld der konfoderierten Sadstaston $\ldots \ldots \ldots \ldots \ldots \ldots \ldots \ldots \ldots .214$ 


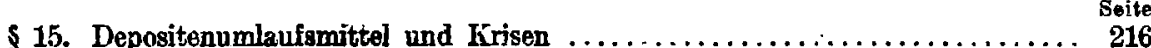

\$ 16. Besondere Krisen ..................................... 218

\$ 17. Depositenumlaufggeschwindigkeit und Krisen $\ldots \ldots \ldots \ldots \ldots \ldots \ldots \ldots$. 220

\$18. Zusammenfassung $\ldots \ldots \ldots \ldots \ldots \ldots \ldots \ldots \ldots \ldots \ldots \ldots \ldots \ldots \ldots, 223$

XII. Kapitel.

Statistiken der letaten Jahre.

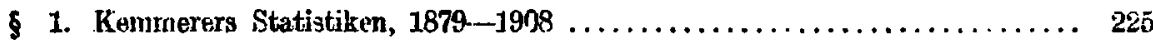

\$ 2. Nere Srhätzungen von $G$ und $G^{\prime}, 1896-1909 \ldots \ldots \ldots \ldots \ldots \ldots \ldots \ldots, 228$

$\S$ 3. Neua Schätzungen von $\sigma^{\prime} U^{\prime}$ und $U^{\prime}, 1896-1909 \ldots \ldots \ldots \ldots \ldots \ldots \ldots 230$

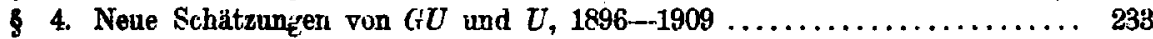

5. Schätzungen von $H$ und $P, 1896-1909 \ldots \ldots \ldots \ldots \ldots \ldots \ldots \ldots \ldots . \ldots \ldots, 237$

$\S$ 6. Direkte und indirekte Bertchnung von $P \ldots \ldots \ldots \ldots \ldots \ldots \ldots \ldots, 238$

7. Korrektur von Abweichungen $\ldots \ldots \ldots \ldots \ldots \ldots \ldots \ldots \ldots \ldots \ldots \ldots, 243$

8. Die Endergebnisse .................................... 248

月. Dis relative Wichtigkeit preissteigernder Ursachen ................ 251

10. Einflub vorausgehender Ursachen, z. B. der Zölle usw. .............. 255

11. Resultate and Nebenergebnisse des XII. Kapitels ................. 258

XIII. Kapitol.

Das Problem, die Kaufkraft stabiler zu gestalten.

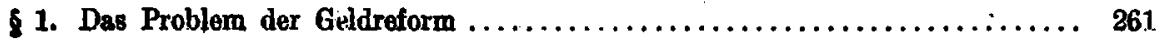

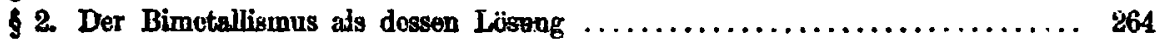

\$ 3. Vorschläge anderer Löstngen $\ldots \ldots \ldots \ldots \ldots \ldots \ldots \ldots \ldots \ldots \ldots \ldots \ldots, 269$

\$ 4. Die Tabellenwährung $\ldots \ldots \ldots \ldots \ldots \ldots \ldots \ldots \ldots \ldots \ldots \ldots \ldots \ldots \ldots, 272$

§ 5. Der Vorschlag des Verfassers $\ldots \ldots \ldots \ldots \ldots \ldots \ldots \ldots \ldots \ldots \ldots \ldots \ldots \ldots, 276$

6. Zusammenfassung und BchluB $\ldots \ldots \ldots \ldots \ldots \ldots \ldots \ldots \ldots \ldots \ldots, 284$

\section{Anhang.}

\section{Anhang zam II. Kapitel.}

$\$ 1$ (zum II. Kapitel, \$ 3). Der Begriff eines Mittelwertes $\ldots \ldots \ldots \ldots \ldots \ldots \ldots 286$

$\$ 2$ (zum 11. Kapitel, § 5). Der Begriff „Umlaufsgesch windighrit" .......... 289

$\$ 3$ (zum II. Kapitel, \$ 5). Anordnung der $p, Q$ und $p Q \ldots \ldots \ldots \ldots \ldots \ldots$ 291

$\$ 4$ (zum II. Kapitel, \$5). Anordnung der $a, g$ und $U \ldots \ldots \ldots \ldots \ldots \ldots 294$

§5 (zum I1. Kapitel, § 5). Der Mü̈nzenilbertragungsbegriff der Geschwindigkeit und der Begriff Umsatzzeit ................................. 298

\$ 6 (zum II. Kapitel, \$ 5). Algebraische Darlegung der Verkehrsgleichung ..... 299

$\$ 7$ (zum II. Kapitel, 5), $P$ mut eine spezifische Form des Durehschnittes sein, um gich direkt wiv $Q$ und $V$ und umgekehrt wie dic $Q$ zu verändern ......300 
$\$ 1$ (zun III. Kapitel, \$2). Anordnung dor $k$ und $r \ldots \ldots \ldots \ldots \ldots \ldots \ldots \ldots$ 30.

\$2 (zum III. Kapitel, $\$ 4$ ). Algebraische Jarlegung der Verkehrsgleichung nit EinschluB der Jepositenumlaufsmittel $\ldots \ldots \ldots \ldots \ldots \ldots \ldots \ldots \ldots \ldots \ldots .30 \%$

\section{Arhang zum V. Kapitel.}

\$ 1 (zum V. Kapitel; \$ 5). Wirknng des Zejtkredits auf die Verkehrogleichnng... 30

\section{Anhang zum VI. Kapitel.}

\$ 1 (zum VI. Kapitel, $\$ 1$ ). Die durch den internationalen Handel irforderliche Modifikation der Verkehrsgleichung $\ldots \ldots \ldots \ldots \ldots \ldots \ldots \ldots \ldots \ldots \ldots \ldots \ldots, 305$

Anhang zum VII. Kapitel.

\$ 1 (2um VII. Kapitel, \$2). Geldsurrogate angleich anderen Ersatzn itteln .... 30 o $\$ 2$ (zum VIJ. Kapitel, § 2). Grenzen de? Verniltuisse, innerhalb deren dir Bimet:allismus möglich ist

\section{Anhang zum VIII. Kapitel.}

\$1 (zum VIII. Kapitel, §6). Umsatzstatistik an der Yale Universität ....... 311

$\S 2$ (zum VIII. Kapitel, \& 8). Gegenüberstellung von vier Warengattungen .... 314

\section{Anhang zum X. Kapitel.}

\$ 1. Jede Forn der Indexziffer für Preise umfaßt eino korrelative Form der Indexziffer für Quantitäten................................... $\mathbf{3 1 6}$

5 2. Indexziffern ftir Preise kommen ebenso wie die Indexziffern für Quantitäten in antithetischen Paren vor $\ldots \ldots \ldots \ldots \ldots \ldots \ldots \ldots \ldots \ldots \ldots \ldots \ldots \ldots, 321$

5. Allgemeine Bedeutung der $p$ und $Q \ldots \ldots \ldots \ldots \ldots \ldots \ldots \ldots \ldots \ldots \ldots$

5. Ubersicht fiber die 44 Formeln als Kolonnentitel der Tabelle . . . . . . . . 324

5. Ubersicht über die als Titel der Tabeilenreiben figurierenden acht Proben ... 330

6. Der innere Tcil dar Tabelle, insbesondere Kolonne 11............... 338

7. Vergleich der 44 Formeln ............................. 346

8. Grtinde für die Bevorzugung dor Mittelzah] für praktische Zwocke ....... 353

9. Kurze ansammenfassende tbersicht $\ldots \ldots \ldots \ldots \ldots \ldots \ldots \ldots \ldots \ldots \ldots \ldots \ldots . \ldots 56$

Anhang zam XII. Kapitel.

$\$ 1$ (zum Xfl. Kapitel, \$1). Kemmerers Bèrechnungen . ............... $\mathbf{3 5 7}$

$\S 2$ (sum XII. Kapitel, $\S 2$ ). Methode zur Berechnang von $(\xi \ldots \ldots \ldots \ldots \ldots . \ldots 59$

$\$ 3$ (zum XIl. Kapitel, § 2). Methode zux Berechnung von $\xi^{\prime} \ldots \ldots \ldots \ldots . .362$

4 (rom XII. Kapitel, \& 3). Methode zur Berechnung ron $G^{\prime} D^{\prime}$ foir die Jahre

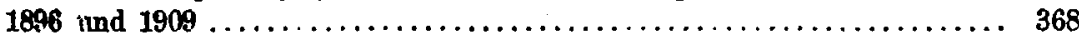

$\$ 5$ (2nm XII. Kapitel, \$ 3). Methode zur Berechnong von $G^{\prime} C^{\prime}$ fir die Jabre $1897-1908$. .......................................... 373 
$\$ 6$ (zum XII. Kapitel, \$4). Allgamein anwendbare Formel zur Berachooug

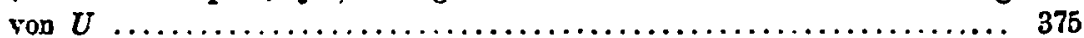

$\S 7$ (zum XII. Kapitel, $\$ 4$ ). Anwendung áer Formel zur Berechnung von $U$ far die Jahre 1896 und $1909 \ldots \ldots \ldots \ldots \ldots \ldots \ldots \ldots \ldots \ldots \ldots \ldots \ldots \ldots$

\$ 8 (zam XII. Kapitel, \$ 4). Interpolation der $U$-Werte für die Jahre

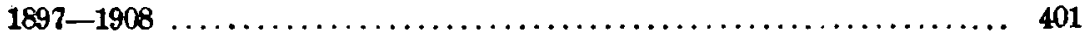

§ 9 (zum XII. Kapitel, § 5). Methodo zur Berechnung von $H \ldots \ldots \ldots \ldots \ldots 402$

$\$ 10$ (zum XII. Kapitel, $\S 5$ ). Methode zur Berechnung von $P \ldots \ldots \ldots \ldots \ldots 409$

$\$ 11$ (zom XII. Kapitel, \$ 7). Gegenseitige Anglejchungen der berechnetrn Werte

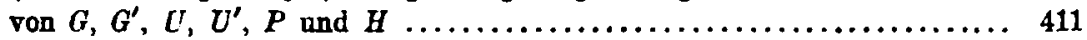

$\$ 12$ (zum XIL. Kapitel, § 8). Kredit- und Bargeldgeschäfte. Vergleich mit Kinleys Schatzungen $\ldots \ldots \ldots \ldots \ldots \ldots \ldots \ldots \ldots \ldots \ldots \ldots \ldots \ldots \ldots, 413$

Nachtrag zur zweiten (amerikanischen) Auflage................... 415

Anhang zur zweiten (amerikanischen) Anflage über die „Wertstabilisierung des Dollars" $\ldots \ldots \ldots \ldots \ldots \ldots \ldots \ldots \ldots \ldots \ldots \ldots \ldots \ldots \ldots \ldots \ldots \ldots \ldots^{416}$ 